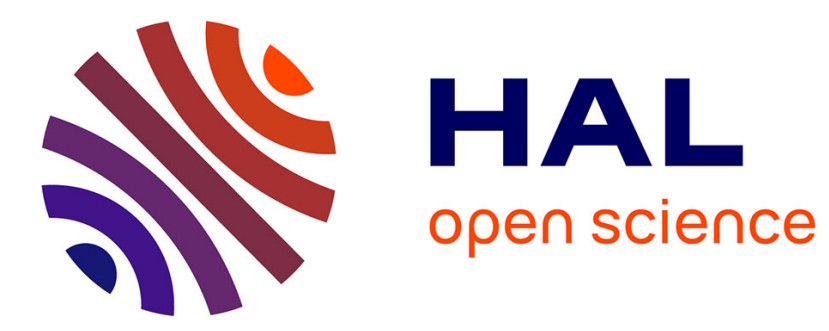

\title{
Tunable phonon nanocapacitor built by carbon schwarzite based host-guest system
}

Zhongwei Zhang, Shiqian Hu, Qing Xi, Tsuneyoshi Nakayama, Sebastian Volz, Jie Chen, Baowen Li

\section{- To cite this version:}

Zhongwei Zhang, Shiqian Hu, Qing Xi, Tsuneyoshi Nakayama, Sebastian Volz, et al.. Tunable phonon nanocapacitor built by carbon schwarzite based host-guest system. Physical Review B, 2020, 101, 10.1103/physrevb.101.081402 . hal-03011202

\section{HAL Id: hal-03011202 https://hal.science/hal-03011202}

Submitted on 18 Nov 2020

HAL is a multi-disciplinary open access archive for the deposit and dissemination of scientific research documents, whether they are published or not. The documents may come from teaching and research institutions in France or abroad, or from public or private research centers.
L'archive ouverte pluridisciplinaire HAL, est destinée au dépôt et à la diffusion de documents scientifiques de niveau recherche, publiés ou non, émanant des établissements d'enseignement et de recherche français ou étrangers, des laboratoires publics ou privés. 


\title{
Tunable phonon nanocapacitor built by carbon schwarzite based host-guest system
}

\author{
Zhongwei Zhang, ${ }^{1,2}$ Shiqian Hu, ${ }^{1,2}$ Qing Xi, ${ }^{1,2}$ Tsuneyoshi Nakayama, ${ }^{1,2,3}$ Sebastian Volz, ${ }^{2,4}$ Jie Chen $\odot,{ }^{1,2,}$ and Baowen $\mathrm{Li}^{5}$ \\ ${ }^{1}$ Center for Phononics and Thermal Energy Science, School of Physics Science and Engineering, \\ Tongji University, 200092 Shanghai, People's Republic of China \\ ${ }^{2}$ China-EU Joint Lab for Nanophononics, Tongji University, 200092 Shanghai, People's Republic of China \\ ${ }^{3}$ Hokkaido University, Sapporo 060-0826, Japan \\ ${ }^{4}$ Laboratory for Integrated Micro and Mechatronic Systems, CNRS-IIS UMI 2820, University of Tokyo, \\ 4-6-1 Komaba, Meguro-ku, Tokyo 153-8505, Japan \\ ${ }^{5}$ Department of Mechanical Engineering and Department of Physics, University of Colorado, Boulder, Colorado 80309, USA
}

(Received 6 July 2018; revised manuscript received 16 January 2020; accepted 22 January 2020; published 7 February 2020)

\begin{abstract}
The ability to control monochromatic and coherent phonons is crucial for many phonon-related applications but remains very challenging. Here, we demonstrate a promising phonon nanocapacitor for storing and emitting phonons, built by the carbon schwarzite based host-guest system (HGS). This phonon nanocapacitor takes advantage of the inherently strong phonon confinement of the hybridized modes in HGS. The monochromaticity and coherence of the stored phonons are well demonstrated via the ultralong phonon lifetime and coherent time. More interestingly, the frequency of the nanocapacitor is widely tunable from gigahertz to several terahertz by engineering the host-guest interaction. Finally, the stored phonons with different polarizations can be emitted separately with the application of uniaxial strain along a particular direction. This work may provide new opportunities for studying the coherent wave effect of phonons.
\end{abstract}

DOI: 10.1103/PhysRevB.101.081402

Introduction. Manipulations of phonons are of primary importance for the exploration of the wave nature of phonons, atomic imaging, and other phononic related fields [1-4]. For example, the phonon is demonstrated as a new carrier to control and exchange information $[2,5,6]$, where the monochromaticity and coherence of phonons are crucial factors. To achieve this, various optomechanical and electromechanical systems have been developed, including the isolated trapped ions system [7], two-level systems [8], and other mechanical oscillators [1,9].

The phonon interference has been used for phononic control, for instance, the subterahertz (THz) acoustic nanocavities based on GaAs/AlAs [10] and the phonon nanocapacitor based on defective silicon for specific $\mathrm{THz}$ phonons [11]. However, the state-of-the-art studies indicate that the manipulation of phonons still encounters notable challenges: (i) Few approaches allow for controlling monochromatic phonons, especially for the ultrahigh $\mathrm{THz}$ frequency. (ii) Only specific phonons with fixed frequency can be controlled in various systems [11,12]. (iii) The coherence is difficult to maintain over long distances $[9,13]$. Therefore, the development of a tunable phonon capacitor is highly desirable.

Host-guest systems (HGSs), such as clathrates, perovskites, and skutterudites [14-16], are usually favorable for thermoelectrics and have received extensive attention. Due to the weak interactions with the host cages, the guest atoms in HGSs possess isolated rattling, which results in the hybridized

*jie@tongji.edu.cn phonon modes with strong confinement and flat band structure. As an inherent feature of HGSs, the unique phonon confinement of the hybridized mode provides a novel platform to control monochromatic phonons $[14,17]$. More interestingly, the frequency of hybridized modes $\left(\omega_{h}\right)$ generally depends on the mass of the guest atoms and the coupling strength with host cages, exhibiting a strong tunability [18-20].

In this work, by utilizing a carbon schwarzite based HGS, we demonstrate a tunable phonon nanocapacitor for storing and emitting monochromatic and coherent phonons. The coherence of the stored phonon is discussed based on the classical coherence function. By considering the rattling motions of guest atoms, we also emphasize the importance of phonon anharmonicity for the phonon nanocapacitor. Our work may provide new opportunities to study thermal transport in nanophononic crystals $[5,21,22]$.

The atomic structure of the phonon nanocapacitor is shown in Fig. 1(a). It is composed of two homogeneous parts: the host cage at the center and HGS layers at two sides. The host cage is a carbon schwarzite structure with a large void space [23], analogous to the empty clathrates [24]. We use classical molecular dynamics (MD) simulations to study the dynamics for the phonon storage and emission processes. MD simulations have been widely used in literature studies on the coherent phonon transport in various superlattice structures $[13,25-27]$. The covalent $\mathrm{C}-\mathrm{C}$ bond in the host cage is modeled by the optimized Tersoff potential [28], and the nonbonded interaction between the host cage and guest atom is modeled by the Lennard-Jones (LJ) potential (see Table S1 in Ref. [29]), which can well capture the "on-center" and "off-center" rattling behaviors in HGSs [20,30]. All MD 


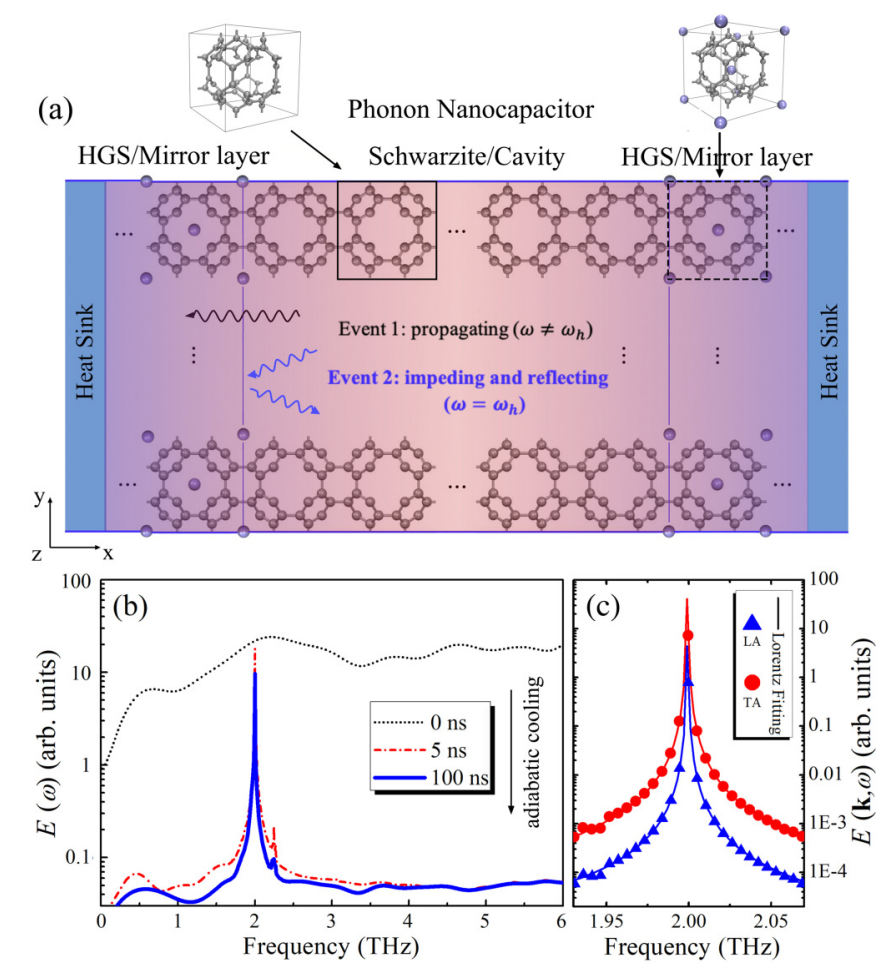

FIG. 1. HGS based phonon nanocapacitor for phonon storage. (a) Atomistic structure for the phonon nanocapacitor composed of full HGS (mirror layers) at the two sides and the carbon schwarzite cavity in the center. At both ends, the phonon nanocapacitor is coupled to heat sinks for applying adiabatic cooling along the length direction. The gray and blue atoms are $\mathrm{C}$ atoms in the host cage and guest atoms, respectively. The upper plane figures show the perspective views of the unit cell for each part. (b) $E(\omega)$ in the cavity for various time snapshots after applying adiabatic cooling from $600 \mathrm{~K}$. (c) $E(\mathbf{k}, \omega)$ for the stored longitudinal acoustic (LA) and transverse acoustic (TA) phonons (symbols), and the Lorentzian fitting (solid line) after $100 \mathrm{~ns}$ of adiabatic cooling. Due to the degeneracy, only one TA mode is shown. The dimensions of the nanocapacitor are $L_{x}=19.6 \mathrm{~nm}$, and $L_{y}=L_{z}=3.9 \mathrm{~nm}$, with three unit cells of mirror layers on each side.

simulations are performed by using the LAMMPS package [31] with a timestep of $0.35 \mathrm{fs}$.

Phonon storage. We first consider the HGS with on-center rattling motion by using the case I LJ parameters (Table S1 in Ref. [29]) and fixed atom mass (Table S2 in Ref. [29]). Under this condition, the highest frequency for acoustic phonon in the host cage is $\omega_{\max } \approx 7.8 \mathrm{THz}$, and the hybridized mode $\omega_{h} \approx 2 \mathrm{THz}$ in HGS (see Fig. S1 in Ref. [29]). The whole system is initially equilibrated with a Langevin thermostat at a high temperature of $600 \mathrm{~K}\left(>2 \pi \hbar \omega_{\max } / k_{B} \sim 375 \mathrm{~K}\right)$, ensuring the full excitation of acoustic phonons in the cage. Afterwards, we remove the high-temperature thermostat and apply the low-temperature heat sinks at two ends to simulate the cooling process [11], as shown in Fig. 1(a). During the cooling process, the temperature difference between cavity and heat sinks leads to the directional propagation of phonon energy, and the phonon energy spectrum in the cavity $E(\omega)$ is recorded (see Sec. IV in Ref. [29]). Compared to the wide-frequency spectrum in the initial state [dotted line in
Fig. 1(b)], only one dominant peak around $2 \mathrm{THz}$ persists even after a long time (100 ns) of continuous cooling [solid line in Fig. 1(b)], demonstrating the feasibility of storing a monochromatic phonon in the cavity.

In other words, except for the phonons with frequency close to $\omega_{h}$, the majority of phonons escaped from the cavity after the cooling process. We have further verified that the successful storage of monochromatic phonons in the nanocapacitor is quite robust for various initial temperatures and system sizes. The increase of the initial temperature or the cavity/HGS length results in a longer escape time for the majority of phonons, which is typically within $100 \mathrm{~ns}$ for various cases (see Sec. V in Ref. [29]).

It should be noted that the storage of monochomatic phonons in the cavity is different from the Bose-Einstein condensation phenomenon where the boson particles condensate to the ground state (with the lowest eigenfrequency) when cooling the system below a critical transition temperature $T_{c}$. In our study, however, the stored single-frequency mode in the cavity is not the ground state, but is the hybridized mode whose frequency is higher than the ground-state frequency and is highly tunable via the host-guest interaction. Furthermore, the estimated $T_{c}$ in our system is $2.96 \mathrm{~K}$ (see Sec. VIII in Ref. [29], and also Ref. [32] therein), which is on the same order of magnitude compared to the literature result [33]. The recorded energy spectrum in the cavity reveals that an obvious peak around $\omega_{h}$ already appears at $t=20$ ps during the cooling process (Fig. S8 in Ref. [29]), for which the averaged temperature of the cavity is $165.9 \mathrm{~K}$, much higher than the estimated $T_{c}$. With further cooling, the averaged temperature of the cavity drops and the peak around $\omega_{h}$ becomes more distinct. Therefore, the absolute value of low-temperature heat sink used in our simulation will only change the monochromaticity of the stored phonon, but will not change the working mechanism of our proposed phonon nanocapacitor.

This phonon storage process can be understood based on the interfacial phonon transmission $(\Theta)$ between the mirror and cavity parts as described by the acoustic mismatch model [34]

$$
\Theta(\omega)=\frac{4 \rho^{m} v_{g}^{m}(\omega) \cos \theta^{m} \rho^{c} v_{g}^{c}(\omega) \cos \theta^{c}}{\left[\rho^{m} v_{g}^{m}(\omega) \cos \theta^{m}+\rho^{c} v_{g}^{c}(\omega) \cos \theta^{c}\right]^{2}},
$$

where $v_{g}$ is the phonon group velocity, $\rho$ is the mass density, $\cos \theta$ is the directional cosine (see Fig. S2 in Ref. [29]), and the superscripts $m$ and $c$ indicate the mirror and cavity parts, respectively. As depicted in Fig. 1(a), two types of phonon transport events take place: (1) For phonons with $\omega \neq \omega_{h}$, since the group velocity is nonzero in both the mirror and cavity parts, this leads to a nonzero transmission coefficient at the interface according to Eq. (1), so that these phonons in the cavity can propagate into HGS given enough time; (2) For phonons with $\omega=\omega_{h}$, the flat band of hybridized modes (zero group velocity) results in the completely forbidden phonon transmission $\left[\Theta\left(\omega_{h}\right)=0\right]$ from the cavity to HGS. Therefore, HGS acts as a mirror (total reflection) for the hybridized modes. At the end of the process, only phonons with hybridized modes frequency are confined in the cavity 
[Fig. 1(b)], achieving the purpose of storing phonons with a particular frequency.

We then discuss the coherent feature of the stored phonons in the cavity. A previous theoretical study by Latour and Chalopin [35] suggests that the phonon coherence length $\left(L_{c}\right)$ is very close to the phonon mean free path $(\Lambda)$ for various frequencies. Therefore, we estimate the coherent length of the stored phonons based on this approximation $L_{c} \approx \Lambda=\tau v_{g}$. To compute the phonon lifetimes $\tau$ for different polarizations, we apply the frequency match method (see Sec. IV in Ref. [29]) and Lorentzian fitting to the energy spectrum as

$$
E(\mathbf{k}, \omega)=\frac{\Xi}{1+\left[\frac{\omega(\mathbf{k})-\omega_{h}}{\gamma}\right]^{2}},
$$

in which $\mathbf{k}$ is the wave vector, $\Xi$ is the peak height, and $\gamma$ is the linewidth obtained from Lorentzian fitting. Due to the small linewidth [Fig. 1(c)], ultralong lifetimes $\left(\tau^{-1}=2 \gamma\right)$ are obtained for the stored phonons $\left(\tau_{L A} \approx\right.$ $3644 \mathrm{~ns}$ and $\tau_{T A} \approx 1568 \mathrm{~ns}$ ). This observed lifetime is several orders of magnitude higher than the intrinsic phonon lifetimes in typical materials, such as graphene and silicon [36], and is also much longer than the cooling time during the storing process ( $\sim 100 \mathrm{~ns})$. Moreover, such ultralong lifetimes have been verified in the nanocapacitor with another size (see Fig. S4 in Ref. [29]). Combined with the group velocity $\left(v_{g}^{L A}=12.71\right.$ $\mathrm{km} / \mathrm{s}$ and $v_{g}^{T A}=8.19 \mathrm{~km} / \mathrm{s}$ ) from the phonon dispersion (Fig. S3 in Ref. [29]), we obtain the coherent length for the stored phonons as $L_{c}^{L A} \approx 46.28 \mathrm{~mm}$ and $L_{c}^{T A} \approx 12.84 \mathrm{~mm}$. This length scale is much longer than the length of the cavity $(\sim 20 \mathrm{~nm})$, indicating that the phonon coherence is well preserved in our system.

Analogous to the coherence in classical optics, the phonon coherence can be described by the coherence function $C(t)$ as [37]

$$
C(t)=\frac{\left\langle\dot{q}\left(\begin{array}{l}
\mathbf{k} \\
s
\end{array} ; t^{\prime}+t\right) \dot{q}\left(\begin{array}{l}
\mathbf{k} \\
s
\end{array} ; t^{\prime}\right)\right\rangle}{\left\langle\dot{q}\left(\begin{array}{l}
\mathbf{k} \\
s
\end{array} ; 0\right) \dot{q}\left(\begin{array}{l}
\mathbf{k} \\
s
\end{array} ; 0\right)\right\rangle},
$$

where $\dot{q}\left({ }_{s}^{\mathbf{k}} ; t\right)$ is the time-dependent phonon normal mode velocity for the stored phonons (k, s) [see Eq. (S4) in Ref. [29]], and $\langle\cdot\rangle$ denotes the ensemble average. Here, we obtain $\dot{q}\left({ }_{s}^{\mathbf{k}} ; t\right)$ after $100 \mathrm{~ns}$ of cooling process, and then calculate the coherence function for LA phonons. As demonstrated by Latour and Chalopin [35], the spatial coherence function follows $\cos \left(\frac{2 \pi}{\lambda} l\right) e^{-l / l_{c}}$, where $l$ is the spatial length, $\lambda$ is the wavelength, and $l_{c}$ is the coherence length. Similarly, the calculated coherence function shown in Fig. 2(a) follows $\cos \left(2 \pi \omega_{h} t\right) e^{-t / \tau_{c}}$, where $\tau_{c}$ is the coherence time. Due to the limited calculation time, the envelope of $C(t)$ is nearly not decayed within $4500 \mathrm{ps}$. The almost flat envelope of $C(t)$ close to unity indicates the ultrastrong coherence for the stored phonon mode $\omega_{h}$, which agrees very well with the predicted ultralong coherent length.

To further understand how the coherence is established during the cooling process, we record the envelopes of coherence functions for phonon mode $\omega_{h}$ at various time snapshots during the cooling process, as shown in Fig. 2(b). Before the cooling process $\left(t_{0}=0\right)$, the whole system is fully
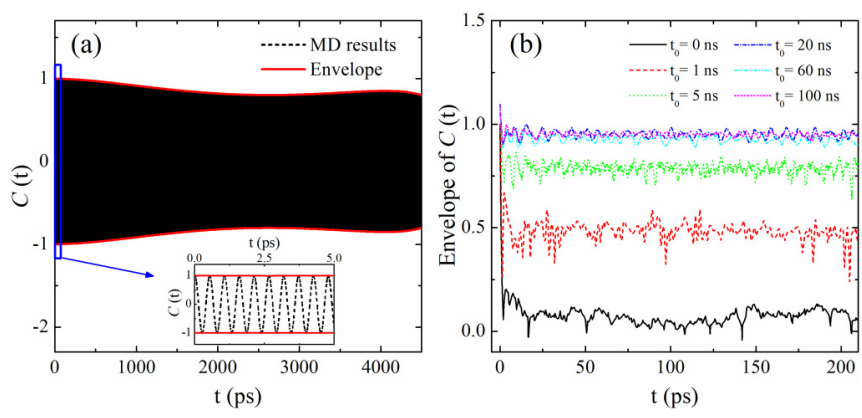

FIG. 2. (a) The coherence function $C(t)$ for the stored phonon mode $\omega_{h}$ after $100 \mathrm{~ns}$ of cooling process. (b) The envelopes of $C(t)$ for the phonon mode $\omega_{h}$ at various time snapshots during the cooling process. Here the LA polarization is used in the calculations for both plots.

thermalized so that the coherence for the phonon mode is quite weak. Correspondingly, the envelope of the coherence function quickly decays to zero at the time $t_{0}=0$. During the cooling process, the degree of coherence for the phonon mode gradually increases over time, as manifested by an increase of the plateau value for the envelope. This plateau value finally saturates to unity after cooling for $20 \mathrm{~ns}$. This observation agrees very well with the convergent monochromatic peak in energy spectrum in the long-time limit, as shown in Fig. 1(b). As the other phonons $\left(\omega \neq \omega_{h}\right)$ continuously escape from the cavity during the cooling process, this essentially reduces the numbers of phonon scattering channels that involve the phonon mode $\omega_{h}$, leading to the increasing degree of coherence for the stored phonon mode $\omega_{h}$ shown in Fig. 2(b).

Different rattling motions of guest atoms, i.e., on-center or off-center, have a critical impact on the phonon dynamics in HGSs $[18,38]$. So far, we have only considered the phonon storage in on-center HGSs [case-I in Fig. 3(a)]. When
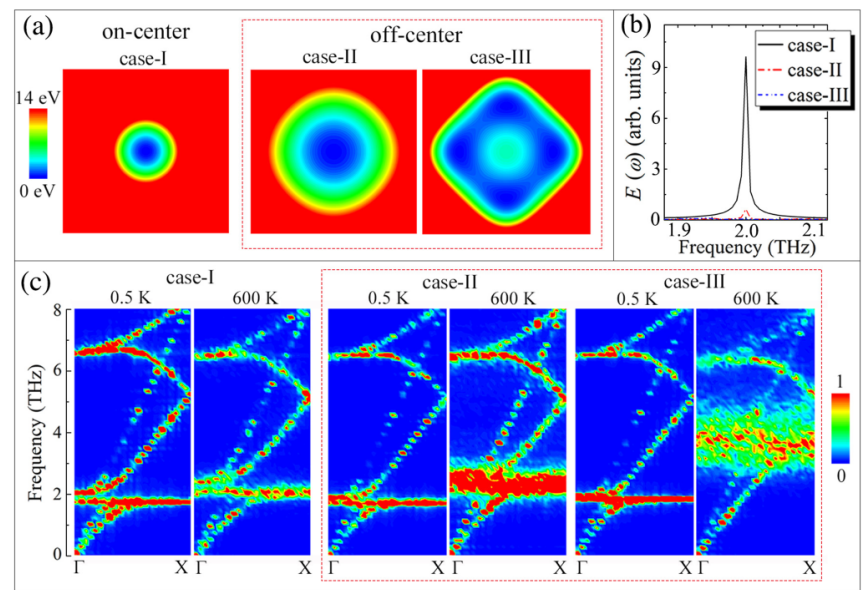

FIG. 3. The impacts of rattling motions on phonon storage. (a) Three types of potential well. case-I: steep potential well for oncenter rattling; case-II and case-III: gentle and asymmetric potential well for off-center rattling, respectively. Here the gentle potential well has strong phonon anharmonicity, and exhibits off-center rattling at high temperature. (b) Storage of phonons after cooling. (c) Phonon dispersion at 0.5 and $600 \mathrm{~K}$ for three types of systems. 
repeating the same simulations for the off-center HGSs with strong anharmonicity [case-II and case-III in Fig. 3(a)], we find that the capability of storing monochromatic phonons is immediately destroyed by the off-center motions [Fig. 3(b)]. To understand such difference, we compute the phonon dispersion at finite temperature based on the spectral energy density method [39]. As shown in Fig. 3(c), there is very limited frequency shift in $\omega_{h}$ and negligible band broadening for the hybridized modes at high temperature for the on-center case, due to the weak anharmonicity. As a result, the hybridized mode $\omega_{h}$ can be successfully stored in the cavity during the adiabatic cooling. In contrast, a remarkable blueshift in $\omega_{h}$ and obvious band broadening are observed at elevated temperature for the off-center cases. These changes eventually lead to the dissipation and escape of phonons with frequency $\omega_{h}$ from the cavity via anharmonic phonon scatterings, causing the failure of the phonon storage in the off-center HGS. In real materials, the HGSs with case-I potential well can be achieved by carefully selecting the types of host cages and guest atoms [19,38].

From the above discussion, we find that on-center rattling motion and the minimized broadening of hybridized modes are favorable for the storage of monochromatic and coherent phonons in HGS based nanocapacitors. In a typical HGS, the upper and lower bound frequencies of the hybridized modes, $\omega_{u b}$ and $\omega_{l b}$, are given by [18]

$$
\omega_{u b}=\frac{1}{2 \pi} \sqrt{\frac{(m+M) K_{e}}{m M}} ; \quad \omega_{l b}=\frac{1}{2 \pi} \sqrt{\frac{4 m f+(m+M) K_{e}-\sqrt{\left[4 m f+(m+M) K_{e}\right]^{2}-16 m M K_{e} f}}{2 m M}} .
$$

Herein, $m$ and $M$ are the mass of the guest atom and the whole host cage, respectively. $K_{e}$ and $f$ are the effective force constants between guest atom and host cage, and between nearest-neighbor cages, respectively. The detailed derivation of this equation is included in Ref. [29]. By plugging in the simulation parameters (Table S2 in Ref. [29]), Eq. (4) predicts $\omega_{u b}=2.05 \mathrm{THz}$ and $\omega_{l b}=1.98 \mathrm{THz}$, which are quantitatively in good agreement with the results from lattice dynamics calculations $\omega_{u b}=2.12 \mathrm{THz}$ and $\omega_{l b}=1.98 \mathrm{THz}$ [Fig. S1(b) in Ref. [29]].

Tunability of phonon nanocapacitor. The feature of minimized broadening in the hybridized mode requires $\omega_{u b} \approx \omega_{l b}$. Such condition can be satisfied in the carbon schwarzite based HGS, as the large cage structure always ensures $M \gg m$. In this case, the frequency of hybridized modes $\omega_{h}$ can be simplified as

$$
\omega_{h} \approx \frac{1}{2 \pi} \sqrt{\frac{K_{e}}{m}} .
$$

In a $\mathrm{LJ}$ model system, we further introduce a dimensionless scaling factor $\eta$ to unify the change of $m$ or $K_{e}$, i.e., $m=m_{0} / \eta$ or $K_{e}=\eta K_{0}$.

The feasibility of phonon storage for different frequencies is first studied by tuning $K_{e}$ with $\eta$. The well preserved highintensity peaks [Fig. 4(a)] and quite small linewidth [inset in Fig. 4(b)] demonstrate the successful storage of monochromatic phonons in a wide range of frequencies. Furthermore, we carry out first-principles calculations to explore the tunability in real materials where $m$ and $K_{e}$ are simultaneously varied [40] (see Sec. VII in Ref. [29], and also Refs. [41-43] therein). By substituting the guest atoms in HGS, $\omega_{h}$ can be gradually tuned from gigahertz to several terahertz [Fig. 4(b)]. Therefore, highly tunable phonon storage can be achieved in such HGS based nanocapacitor by engineering the interactions between the host cage and guest atoms.

Phonon emission. The phonon emission is another important process for the application of phonon nanocapacitors. In HGSs, the phonon confinement of hybridized modes can be manipulated by the external strains, which allows for the emission of phonons stored in the cavity. To quantify the change in the degree of confinement, we compute the participation ratio (PR) for each phonon mode $\lambda$ as [44]

$$
\operatorname{PR}_{\lambda}=\frac{\left(\sum_{i}^{N} \frac{\sum_{\alpha} \varepsilon_{i \alpha, \lambda}^{*} \varepsilon_{i \alpha, \lambda}}{M_{i}}\right)^{2}}{N \sum_{i}^{N} \frac{\left(\sum_{\alpha} \varepsilon_{i \alpha,}^{*} \varepsilon_{i \alpha, \lambda}\right)^{2}}{M_{i}^{2}}}
$$

where $N$ is the total number of atoms, and $\varepsilon_{i \alpha, \lambda}$ and $M_{i}$ are the $\alpha$ th $(x, y, z)$ eigenvector component of the eigen-mode $\lambda$ and the mass for the $i$ th atom, respectively.

In the pristine (strain-free) HGS, the phonons close to $\omega_{h}$ are completely localized $(\mathrm{PR}=0)$ [inset in Fig. 5(a)], which are triplet degenerated and behave as a "locon" [45]. Therefore, three phonon polarizations are simultaneously stored in the cavity. Interestingly, each polarization can be emitted separately by applying a small amount of uniaxial strain along a particular direction. For instance, when applying $0.5 \%$ of uniaxial tensile strain along the [100] direction, the LA branch shifts to a notably lower frequency, while the other two branches are unchanged [Fig. 5(a)]. This frequency shift causes a nonzero PR value for LA phonons at the originally stored frequency $\left(\omega_{h} \approx 2 \mathrm{THz}\right)$, which results in a nonzero

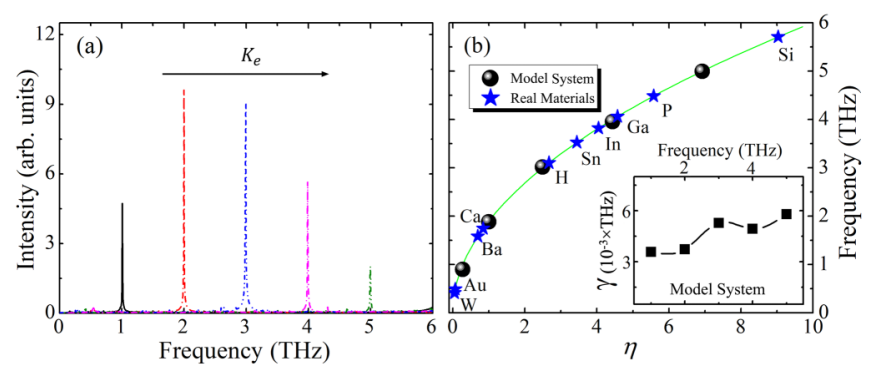

FIG. 4. Frequency tunability of the phonon nanocapacitor. (a) The stored $E(\omega)$ peaks at different frequencies after $100 \mathrm{~ns}$ of cooling. (b) Frequencies of stored phonons as a function of scaling factor $\eta$ in the model system from MD simulations and in real materials from first-principles calculations, respectively. The solid line shows the $\omega_{h} \sim \sqrt{\eta}$ law. The inset in (b) is the linewidth $\gamma$ for the stored phonons in (a). 

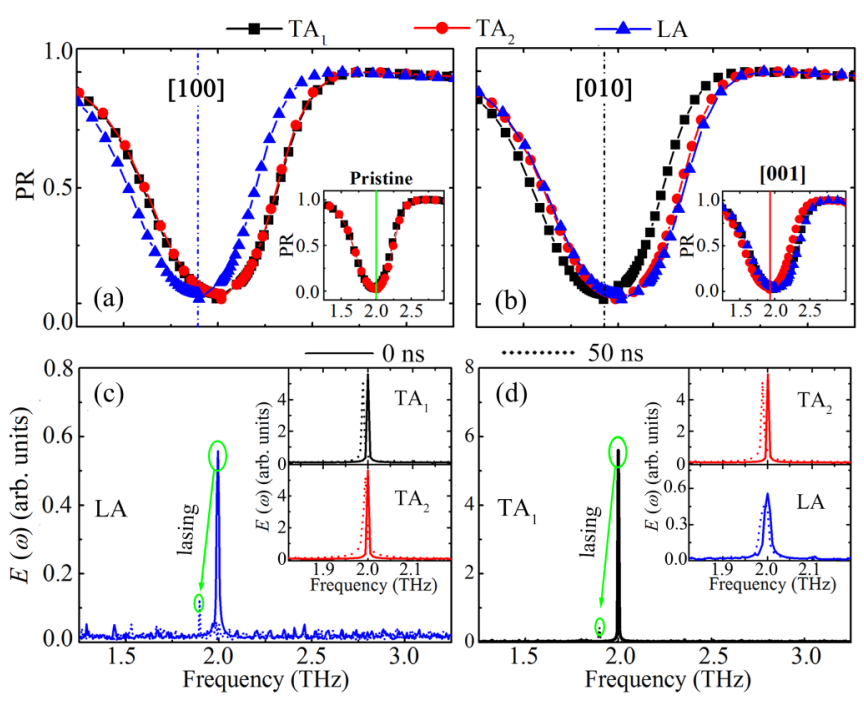

FIG. 5. Phonon emission process. (a) and (b) show the participation ratio (PR) of three acoustic branches in HGS under $0.5 \%$ of uniaxial tensile strain along the [100] and [010] directions, respectively. The insets in (a) and (b) are the PR for the pristine (strain-free) and strained HGS along the [001] direction, respectively. (c) and (d) show the energy spectrum in the cavity for the LA and $\mathrm{TA}_{1}$ phonons, respectively, before (solid line) and after (dashed line) applying strain. The insets in (c) and (d) show the other two polarizations are almost unaffected by the strain.

$\Theta\left(\omega_{h}\right)$ and thus the emission of a LA phonon in the cavity [Fig. 5(c)]. Meanwhile, two TA phonons remain stored in the cavity as their frequencies are unchanged. This procedure is also valid for emitting the transverse mode [Figs. 5(b) and 5(d)]. Such phonon emission process can be realized by periodically pumping the stress using a piezoelectric transducer [9].
The carbon schwarzite structure has only one type of cage structure with tunable Gaussian curvature [23], and relatively weak anharmonicity, making it possible to store monochromatic phonons. Moreover, the insertion of the guest atom into the schwarzite cage only leads to negligible size change of the cage structure, due to the strong stiffness of the covalently bonded carbon schwarzite cage and the weak van der Waals interaction between the cage and the guest atom. Therefore, there is almost no strain between the HGS and central cavity. These unique advantages make the carbon schwarzite an ideal candidate for building the phonon nanocapacitor. For other HGSs, such as clathrates [46,47], they may have multiple flat modes and strong phonon anharmonicity, due to the complex crystal symmetry and different types of cage structures, which may pose challenges for the storage of monochromatic phonons. In addition, the local strain generally can exist in real experiments, due to the lattice mismatch between the HGS and cavity, which will cause a frequency shift of the eventually stored phonons compared to the ideal strain-free case.

Summary. We have proposed a phonon nanocapacitor for storing and emitting monochromatic and coherent phonons, by utilizing the inherent hybridized modes in the carbon schwarzite based HGS. Our proposed nanocapacitor shows a wide-frequency tunability, from gigahertz to several terahertz. The ultralong coherent time of the stored phonon is demonstrated via the classical coherence function. The phonon nanocapacitor can be synthesized via the solid-state reaction process [48], and the nanocapacitor with different guest atoms would also be achieved by varying the stoichiometric mixtures during the reaction [49].

Acknowledgments. This project is supported in part by the grants from the National Natural Science Foundation of China (Grant No. 11890703), and the Science and Technology Commission of Shanghai Municipality (Grants No. 19ZR1478600, No. 18JC1410900, and No. 17ZR1448000). J. C. acknowledges support from the National Youth 1000 Talents Program in China.
[1] J. D. Cohen, S. M. Meenehan, G. S. MacCabe, S. Gröblacher, A. H. Safavi-Naeini, F. Marsili, M. D. Shaw, and O. Painter, Nature (London) 520, 522 (2015).

[2] A. H. Safavi-Naeini, T. P. Alegre, J. Chan, M. Eichenfield, M. Winger, Q. Lin, J. T. Hill, D. E. Chang, and O. Painter, Nature (London) 472, 69 (2011).

[3] M. V. Gustafsson, T. Aref, A. F. Kockum, M. K. Ekström, G. Johansson, and P. Delsing, Science 346, 207 (2014).

[4] J. Ravichandran, A. K. Yadav, R. Cheaito, P. B. Rossen, A. Soukiassian, S. J. Suresha, J. C. Duda, B. M. Foley, C. H. Lee, Y. Zhu, A. W. Lichtenberger, J. E. Moore, D. A. Muller, D. G. Schlom, P. E. Hopkins, A. Majumdar, R. Ramesh, and M. A. Zurbuchen, Nat. Mater. 13, 168 (2014).

[5] N. Li, J. Ren, L. Wang, G. Zhang, P. Hänggi, and B. Li, Rev. Mod. Phys. 84, 1045 (2012).

[6] Y. Chu, P. Kharel, W. H. Renninger, L. D. Burkhart, L. Frunzio, P. T. Rakich, and R. J. Schoelkopf, Science 358, 199 (2017).

[7] K. Vahala, M. Herrmann, S. Knünz, V. Batteiger, G. Saathoff, T. W. Hänsch, and T. Udem, Nat. Phys. 5, 682 (2009).
[8] I. S. Grudinin, H. Lee, O. Painter, and K. J. Vahala, Phys. Rev. Lett. 104, 083901 (2010).

[9] H. Okamoto, A. Gourgout, C. Y. Chang, K. Onomitsu, I. Mahboob, E. Y. Chang, and H. Yamaguchi, Nat. Phys. 9, 480 (2013).

[10] A. Huynh, N. D. Lanzillotti-Kimura, B. Jusserand, B. Perrin, A. Fainstein, M. F. Pascual-Winter, E. Peronne, and A. Lemaitre, Phys. Rev. Lett. 97, 115502 (2006).

[11] H. Han, B. Li, S. Volz, and Y. A. Kosevich, Phys. Rev. Lett. 114, 145501 (2015).

[12] M. Trigo, A. Bruchhausen, A. Fainstein, B. Jusserand, and V. Thierry-Mieg, Phys. Rev. Lett. 89, 227402 (2002).

[13] S. Hu, Z. Zhang, P. Jiang, J. Chen, S. Volz, M. Nomura, and B. Li, J. Phys. Chem. Lett. 9, 3959 (2018).

[14] T. Takabatake, K. Suekuni, T. Nakayama, and E. Kaneshita, Rev. Mod. Phys. 86, 669 (2014).

[15] S. Pailhès, H. Euchner, V. M. Giordano, R. Debord, A. Assy, S. Gomès, A. Bosak, D. Machon, S. Paschen, and M. de Boissieu, Phys. Rev. Lett. 113, 025506 (2014). 
[16] Q. Xi, Z. Zhang, J. Chen, J. Zhou, T. Nakayama, and B. Li, Phys. Rev. B 96, 064306 (2017).

[17] C. Chen, Z. Zhang, and J. Chen, Front. Energy Res. 6, 34 (2018).

[18] T. Nakayama and E. Kaneshita, J. Phys. Soc. Jpn. 80, 104604 (2011).

[19] J. Dong, O. F. Sankey, G. K. Ramachandran, and P. F. McMillan, J. Appl. Phys. 87, 7726 (2000).

[20] Z. Zhang, S. Hu, T. Nakayama, J. Chen, and B. Li, Carbon 139, 289 (2018).

[21] X. Gu, Y. Wei, X. Yin, B. Li, and R. Yang, Rev. Mod. Phys. 90, 041002 (2018).

[22] G. Xie, Z. Ju, K. Zhou, X. Wei, Z. Guo, Y. Cai, and G. Zhang, npj Comput. Mater. 4, 21 (2018).

[23] Z. Zhang, J. Chen, and B. Li, Nanoscale 9, 14208 (2017).

[24] R. Lv, E. Cruz-Silva, and M. Terrones, ACS Nano 8, 4061 (2014).

[25] S. Hu, Z. Zhang, P. Jiang, W. Ren, C. Yu, J. Shiomi, and J. Chen, Nanoscale 11, 11839 (2019).

[26] Y. Wang, H. Huang, and X. Ruan, Phys. Rev. B 90, 165406 (2014).

[27] T. Zhu and E. Ertekin, Phys. Rev. B 90, 195209 (2014).

[28] L. Lindsay and D. A. Broido, Phys. Rev. B 81, 205441 (2010).

[29] See Supplemental Material at http://link.aps.org/supplemental/ 10.1103/PhysRevB.101.081402 for (Sec. I) interaction parameters, (Sec. II) phonon properties for host-guest system, (Sec. III) schematic figure of phonon transmission, (Sec. IV) phonon information calculation methods, (Sec. V) phonon storing process for various initial temperature and system size, (Sec. VI) broadening of hybridized modes, (Sec. VII) first-principles calculation details, and (Sec. VIII) cavity temperature during cooling.

[30] J. Dong, O. F. Sankey, and C. W. Myles, Phys. Rev. Lett. 86, 2361 (2001).

[31] S. Plimpton, J. Comput. Phys. 117, 1 (1995).
[32] L. Pitaevskii and S. Stringari, Bose-Einstein Condensation and Superfluidity (Oxford University Press, New York, 2016), Vol. 164.

[33] O. Misochko, M. Hase, K. Ishioka, and M. Kitajima, Phys. Lett. A 321, 381 (2004).

[34] G. Chen, Phys. Rev. B 57, 14958 (1998).

[35] B. Latour and Y. Chalopin, Phys. Rev. B 95, 214310 (2017).

[36] A. Cepellotti and N. Marzari, Phys. Rev. X 6, 041013 (2016).

[37] G. Xie, D. Ding, and G. Zhang, Adv. Phys. X 3, 719 (2018).

[38] T. Tadano and S. Tsuneyuki, Phys. Rev. Lett. 120, 105901 (2018).

[39] J. M. Larkin, J. E. Turney, A. D. Massicotte, C. H. Amon, and A. J. H. McGaughey, J. Comput. Theor. Nanosci. 11, 249 (2014).

[40] H. Euchner, S. Pailhès, V. M. Giordano, and M. de Boissieu, Phys. Rev. B 97, 014304 (2018).

[41] G. Kresse and J. Furthmüller, Phys. Rev. B 54, 11169 (1996).

[42] O. Hellman, I. A. Abrikosov, and S. I. Simak, Phys. Rev. B 84, 180301(R) (2011).

[43] O. Hellman and I. A. Abrikosov, Phys. Rev. B 88, 144301 (2013).

[44] J. Hafner and M. Krajci, J. Phys.: Condens. Matter 5, 2489 (1993).

[45] J. Fabian and P. B. Allen, Phys. Rev. Lett. 77, 3839 (1996).

[46] T. Tadano, Y. Gohda, and S. Tsuneyuki, Phys. Rev. Lett. 114, 095501 (2015).

[47] P. F. Lory, S. Pailhes, V. M. Giordano, H. Euchner, H. D. Nguyen, R. Ramlau, H. Borrmann, M. Schmidt, M. Baitinger, M. Ikeda, P. Tomes, M. Mihalkovic, C. Allio, M. R. Johnson, H. Schober, Y. Sidis, F. Bourdarot, L. P. Regnault, J. Ollivier, S. Paschen, Y. Grin, and M. de Boissieu, Nat. Commun. 8, 491 (2017).

[48] J. Fulmer, O. I. Lebedev, V. V. Roddatis, D. C. Kaseman, S. Sen, J. A. Dolyniuk, K. Lee, A. V. Olenev, and K. Kovnir, J. Am. Chem. Soc. 135, 12313 (2013).

[49] B. C. Sales, B. C. Chakoumakos, R. Jin, J. R. Thompson, and D. Mandrus, Phys. Rev. B 63, 245113 (2001). 